\title{
Turbulencia económica, violencia y cambios migratorios en la frontera norte de México, 1990-2010
}

\author{
ELMYRA YBÁÑEZ ZEPEDA* \\ RAFAEL ALARCÓN**
}

RESUMEN: El gran crecimiento poblacional en la frontera norte de México que culminó al final del siglo xx se debió, principalmente, a la gran inmigración procedente de otros estados del país. A este proceso le siguió una disminución en el ritmo de crecimiento poblacional debido a la combinación de la reducción de la inmigración y el incremento de la emigración que se observa en la primera década del siglo xxI. Este artículo tiene como objetivo central describir y analizar los cambios ocurridos durante los últimos 20 años en las estructuras por edad de la población de las entidades que colindan con Estados Unidos entre 1990 y 2010. El desempleo, la percepción de violencia e inseguridad por parte de la población que habita en los estados de la frontera norte de México ha impactado la movilidad de las personas afectando la tasa de crecimiento poblacional durante la última década y con consecuencias visibles en la estructura por edad de las pirámides de 2010.

PALABRAS CLAVE: migración, desempleo, inseguridad, cambios demográficos, frontera norte.

\footnotetext{
* Profesora-investigadora del Departamento de Estudios de Población de El Colegio de la Frontera Norte, México.

**Profesor-investigador del Departamento de Estudios Sociales de El Colegio de la Frontera Norte, México.
} 
ABSTRACT: The large population growth in states along the northern border of Mexico at the end of the 20th Century was mainly due to a significant immigration from other states within the country. This process was followed by a drop in the population growth rate that occurred as the result of a combination of reduced immigration and a growth of emigration in the first decade of the 21st Century. The main aim of this article is to describe and analyze the changes in the age structure of the population that have taken place in the past 20 years in the border areas between 1990 and 2010. Unemployment together with the perception of insecurity and violence have had an impact on the mobility of the population living in the Mexican northern border states, with repercussions in the population growth rates over the past decade and its consequences in the age population pyramids by 2010 .

KEY WORDS: Migration, age structure, economic crisis, insecurity, Mexican northern border. 


\section{INTRODUCCIÓN}

7 os estados de la frontera norte de México colindante con Estados Unidos -Baja California, Sonora, Chihuahua, Coahuila, Nuevo León y Tamaulipas - se han caracterizado por presentar una dinámica poblacional y económica distinta al resto del país. La alta concentración urbana de sus municipios, ubicados en los primeros lugares de desempeño económico, les ha permitido disponer de los mejores niveles de bienestar, por lo menos hasta la última década del siglo Xx.

Dichos estados han experimentado transformaciones sociales, económicas y demográficas en un entorno de crecimiento sostenido de la población debido, principalmente, a la alta inmigración procedente del interior del país gracias a la oferta de empleo, la inversión extranjera directa y la apertura comercial (Mendoza, 2010). En este contexto, la frontera norte había sido uno de los polos de atracción poblacional más dinámicos del país.

Entre 1980 y 2000, la tendencia de esta inmigración estuvo enmarcada en un nuevo modelo de migración interna en el cual estos recientes centros de atracción poblacional se convirtieron en regiones dinámicas incorporadas al proceso de globalización a través, por ejemplo, de la instalación de la industria maquiladora. Este tipo de migración interna sustituyó el modelo que se dio durante el periodo de industrialización por sustitución de importaciones entre las décadas de 1940 y 1970 que condujo a una fuerte concentración de población y actividad productiva en los tres grandes centros urbanos del país (Distrito Federal, Guadalajara y Monterrey) y que se vio acompañada de la migración del campo a la ciudad, sobre todo a las tres grandes metrópolis (Chávez Galindo y Lozano Ascencio, 2004).

Sin embargo, en la primera década del siglo XXI, las tendencias de la migración interna muestran un cambio importante en los estados de la frontera norte de México: disminuye la atracción de la población que había tenido en décadas anteriores y en su lugar surgen estados con altas tasas netas de inmigración. Quintana Roo tiene una tasa neta de migración de 19.28 por mil en el periodo 1995-2000 y de 12.65 por mil en el periodo 2005-2010. Asimismo, Baja California Sur, con una tasa neta de migración de 9.01 por 
mil para el periodo 1995-2000, la incrementa a 17.75 por mil en 2005-2010. Estos dos estados se convierten en los principales polos de atracción para la mano de obra tanto en servicios como en la construcción. La fuerte atracción poblacional se debe al fomento del turismo en los nuevos polos dinámicos de la economía nacional que se especializan en el turismo internacional de alto nivel económico.

Este artículo tiene como objetivo central describir y analizar los cambios ocurridos durante los últimos 20 años en las estructuras por edades de la población de las entidades que colindan con Estados Unidos entre 1990 y 2010 como efecto de la disminución de la inmigración y el aumento de la emigración a causa del incremento del desempleo en la región y de la percepción de la inseguridad causada por el crecimiento del número de homicidios.

Se analizan dos posibles causas de los cambios observados. Por una parte, la disminución del flujo de inmigrantes hacia los estados de la frontera norte del país debido al aumento en la tasa de desempleo y al impacto de la crisis económica en Estados Unidos que afecta directamente a la industria maquiladora de exportación (IME) en los estados norteños (Mendoza, 2010). Por otro lado, se examina la pérdida de población en edades productivas y reproductivas a causa de la emigración debido a la caída de los empleos en la IME, y a la percepción de inseguridad y violencia en dichos estados que ha experimentado el crecimiento del número de homicidios (Escalante, 2009).

Las fuentes de información usadas en este trabajo son los Conteos de Población y Vivienda de 1995 y 2005, los Censos de Población y Vivienda de 1990, 2000 y 2010, los datos sobre repatriados del Instituto Nacional de Migración (INM, 2010) y la tasa de homicidios del INEGI (2011), además de la revisión de la literatura sobre los temas que se plantean.

El artículo está dividido en cuatro secciones. En la primera se describen la dinámica y las tendencias económicas de los estados de la frontera norte por lo que se estudia el efecto en la caída de la demanda de empleo en la región a través de la tasa de desempleo entre 2000 y 2008. En la segunda se estudian los efectos que la creciente violencia e inseguridad han tenido en las tasas de crecimiento en esta región mediante las tasas de homicidio entre 1997 y 2010. En la tercera sección se revisan las tasas de inmigración y emigración

$64 \frac{\text { PRIMER SEMESTRE } 2014}{\text { MIGRACIÓN Y DESARROLLO NÚM. } 22}$ 
de los estados de la frontera norte entre 1990 y 2010 para evaluar el impacto social del cambio en los últimos años. Uno de los efectos de la política de inmigración de Estados Unidos en la franja fronteriza ha resultado en el incremento de los migrantes mexicanos repatriados desde la frontera y desde el interior de Estados Unidos. Por esta razón, se cuestiona hasta qué punto estas personas incrementan el volumen de la población fronteriza, por lo que se describen los cambios en el número de repatriados entre 1995 y 2010. En la cuarta se estudia el proceso de transformación que ha tenido el crecimiento demográfico en los estados de la frontera norte a través de las tasas de crecimiento de la población entre 1990 y 2010. Se analizan también las transformaciones en las estructuras por edad de la población mediante la comparación de las pirámides de la población para los estados de Baja California, Sonora, Chihuahua, Coahuila, Nuevo León y Tamaulipas en 1990, 2000 y 2010. Por último, en las conclusiones se discuten los efectos que han tenido el incremento del desempleo en la región y el incremento de la percepción de la inseguridad causada por el crecimiento del número de homicidios en los cambios en las estructuras de la población fronteriza del norte de México.

\section{Aumento en la tAsa de desempleo}

México experimentó un proceso de crecimiento económico desde la última recesión de 1994 y hasta finales de 2008. Particularmente, los estados de la frontera norte tuvieron un mayor dinamismo en dicho crecimiento. Mientras el país creció a una tasa anual de $2.9 \%$, los estados norteños lo hicieron a $4.1 \%$ anual. Lo anterior fue posible gracias a la base industrial y manufacturera ya establecida en la región y a la inversión extranjera directa (Mendoza, 2010: 12).

En cuanto al mercado laboral, durante la década de los noventa hubo un crecimiento del empleo en la industria y la manufactura. Sin embargo, las recesiones económicas impactaron en el crecimiento del empleo en ambos sectores. En el primer quinquenio del siglo XXI, la economía de los estados de 
la frontera norte tuvieron un periodo de crisis al no crecer ni generar los empleos que se demandaban. En la gráfica 1 se muestran las tasas de desempleo entre 2000 y 2010 para México, el promedio para los estados de la frontera norte y para cada uno de ellos.

\section{GRÁFICA 1}

Tasa de desempleo, México y estados de la frontera norte, 2000-2010

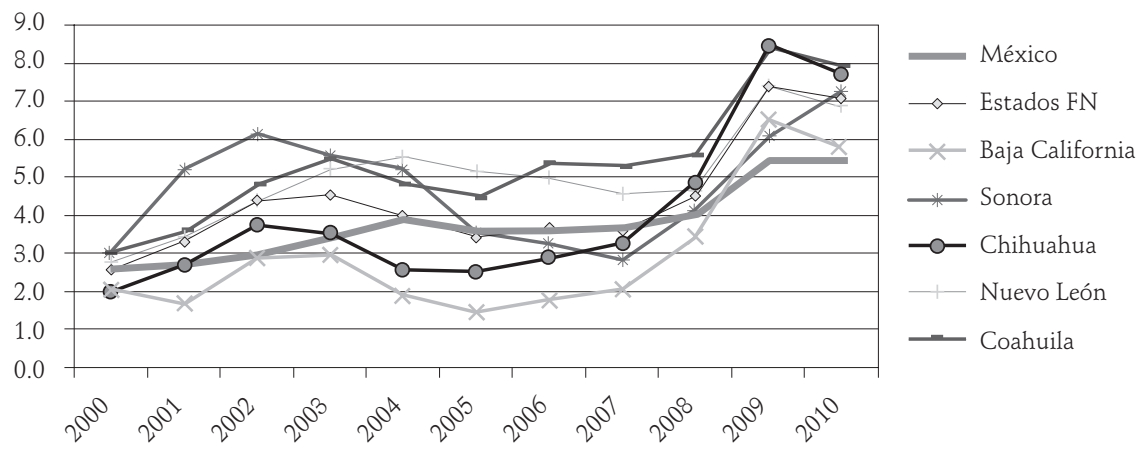

Fuente: Elaboración a partir de Mendoza (2013).

Como puede observarse en la Gráfica 1, Baja California es el único estado que mantiene una tasa de desempleo inferior a la del país hasta 2008. Por su parte, Chihuahua solamente en 2004, 2005 y 2006 muestra este comportamiento. El resto de los estados muestran una tasa de desempleo por encima del nivel nacional. Nuevo León y Coahuila destacan con una tasa superior a la de México durante los diez años considerados. En 2009, los seis estados de la frontera norte tienen un incremento en la tasa de desempleo, el mayor aumento en toda la década y en 2010 muestran una pequeña recuperación, si bien las tasas se mantienen elevadas. Solamente Sonora es el estado que continúa con una tendencia ascendente en la tasa de desempleo.

En 2009 y 2010, la tasa de desempleo en todos los estados norteños fue especialmente alta, lo cual sugiere una crisis en la industria de la manufactura y la disminución de la inversión extranjera directa de la que se había beneficiado esta región del país durante los noventa. La industria manufac-

$66 \frac{\text { PRIMER SEMESTRE } 2014}{\text { MIGRACIÓN Y DESARROLLO NÚM. } 22}$ 
turera sufrió un proceso de desindustrialización frente a la emergencia de China y los países asiáticos, por lo cual los estados de la frontera norte de México perdieron el perfil de especialización que les permitía insertarse de forma exitosa en el mercado estadounidense (Calderón, 2009). A pesar de que México es uno de los principales socios comerciales de Estados Unidos, China ha sobrepasado a las exportaciones mexicanas y desde 2003 ocupa el lugar de México como segundo exportador a Estados Unidos (Mendoza, 2010).

Este cambio en el comportamiento del mercado laboral de los estados fronterizos ha tenido un impacto en la inmigración procedente de otros estados de México y también ha afectado la emigración desde la región fronteriza hacia otras regiones del país en la búsqueda de un bienestar que ya no están encontrando en estos estados.

Además de los cambios descritos anteriormente, existen múltiples factores que podrían explicar las transformaciones en los fenómenos demográficos y su impacto en las tasas de crecimiento y en las estructuras por edad y sexo de la población. Otro de los factores que se ha observado durante la última década tiene que ver con la inseguridad que se percibe y el incremento en la tasa de homicidios en las entidades del norte de México. En el siguiente apartado se revisan las cifras de las muertes por homicidio y las tasas de muertes por homicidio de los estados de la frontera norte de México y se realiza una comparación con otros estados del interior del país con el fin de analizar hasta qué punto el incremento de los homicidios ha contribuido a la disminución de la tasa de inmigración hacia ciertos estados de la frontera norte.

\section{VIOLENCIA, HOMICIDIO E INSEGURIDAD EN LA FRONTERA NORTE}

La percepción del incremento de violencia e inseguridad en México ha motivado su medición. Desde principios de la década de 2000, se planteó la necesidad de generar información al respecto. Según la Sexta Encuesta Nacional sobre Inseguridad (ENSI-2009), Chihuahua ocupaba el segundo lugar con 83\% 
de la población mayor de 18 años que consideraba insegura a su entidad. Baja California ocupaba el cuarto puesto con $76 \%$ considerándolo inseguro. En Tamaulipas fue tan evidente la inseguridad que no fue posible obtener la información debido a problemas durante el operativo de campo ya que personas ajenas a la encuesta se encontraban vigilando a los encuestados (ICESI, 2009).

En la misma encuesta, Nuevo León ocupó el décimo lugar, pues 70\% de los encuestados declaró sentirse inseguro, Coahuila ocupó el diecisieteavo (58\% de los mayores de 18 años declararon sentirse inseguros) y Sonora el puesto número 23 (solamente 47\% dijeron haberse sentido inseguros) (ICESI, 2009).

Esta creciente sensación de inseguridad en los estados de la frontera norte de México tiene que ver también con lo que los medios de comunicación destacan sobre la violencia en dichos estados. Sin embargo, la situación de entidades federativas como Morelos, Nayarit, Michoacán o Guerrero, por mencionar algunos, es mucho más alarmante. Específicamente, Guerrero ha estado durante todo el periodo con tasas de homicidio que lo ubican entre los primeros diez lugares.

Las muertes violentas por homicidio habían disminuido hasta el inicio del presente siglo. Hernández (1989) señala que las muertes violentas en México pasaron de constituir 15.8\% de las muertes en 1980 a 12\% en 2000. En ese año, el homicidio se encontraba en el noveno lugar entre las causas de muerte en México. En 2001 bajó al décimo lugar y se mantuvo en el onceavo lugar entre 2002 y 2007; sin embargo, en 2008 volvió al décimo lugar, en 2009 al sexto lugar y en 2010 ya ocupaba el quinto lugar (Sinais, 2012).

A partir de 2007, el incremento de la violencia y la inseguridad ha modficado los patrones de movilidad de la población. En los últimos años se ha incrementado la percepción de un aumento de homicidios, debido a la difusión de los medios de comunicación (Escalante, 2009). Por ejemplo, el 65\% de los entrevistados en la Novena Encuesta Nacional sobre Inseguridad percibieron su entidad de residencia como insegura. Lo que se comenzó a especular fue que si las condiciones de seguridad pública empeoraban debido a la cada vez mayor presencia de la delincuencia organizada, se reduciría el crecimiento económico debido a que las ciudades fronterizas perderían su atractivo para

$$
68 \frac{\text { PRIMER SEMESTRE } 2014}{\text { MIGRACIÓN Y DESARROLLO NÚM. } 22}
$$


los turistas y para algunos inversionistas, lo que se manifestaría en una disminución del crecimiento económico (Alegría, 2010) y, por ende, disminuiría también el crecimiento poblacional debido a la inmigración.

En la gráfica 2 se muestra la tasa de defunciones con presunción de homicidio para los estados de la frontera norte de México entre 1997 y 2010. Es impactante el incremento de la tasa de homicidio observada en el estado de Chihuahua a partir de 2008 debido especialmente a los casos de Ciudad Juárez y la ciudad de Chihuahua. De acuerdo al Consejo Ciudadano para la Seguridad Pública y Justicia Social A.C. (2012), las diez ciudades más violentas del mundo en 2010 fueron Ciudad Juárez en México; Kandhar, Afganistán; San Pedro Sula, Honduras; Caracas, Venezuela; Chihuahua, México, Tegucigalpa, Honduras; Guatemala, Guatemala; Mazatlán, México; Culiacán, México, y Medellín, Colombia.

Mientras en Sonora y Tamaulipas se aprecia un ligero incremento en la tasa de defunciones con presunción de homicidio, entre 2007 y 2010 en Baja California se incrementa en 2008 y disminuye en 2009 y 2010. Esta tasa en Nuevo León aumenta en 2010.

\section{GRÁFICA 2}

Tasas de defunciones con presunción de homicidio (DPH) por 100,000 habitantes según entidad federativa y año de ocurrencia, 1997-2010

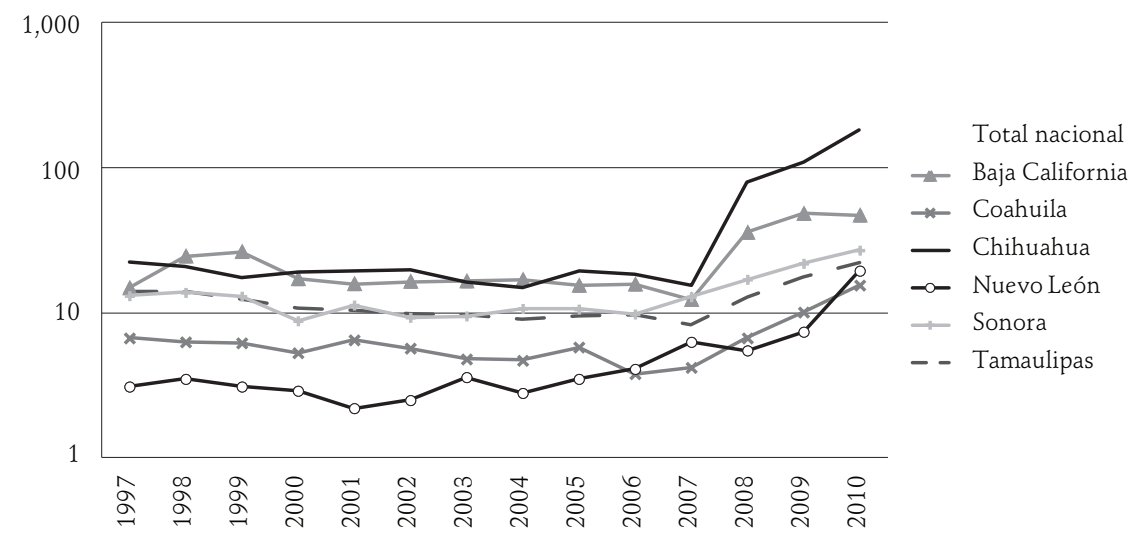

Fuente: CIES, SSP (2012). 
Si bien la tasa de defunciones con presunción de homicidio es elevada en los estados de la frontera norte de México, particularmente en Chihuahua, cabe mencionar que en el contexto nacional son otros estados los que se encuentran en los primeros lugares durante el periodo. En 2000, Guerrero mostraba la tasa más elevada, seguido de Oaxaca y de Jalisco. En ese año, Chihuahua sólo ocupaba el quinceavo puesto. En 2005 se da un cambio notable en los primeros tres puestos que ocupan Sinaloa, Chiapas y Tlaxcala. Chihuahua ocupa el noveno lugar y Tamaulipas el décimo.

En 2008, iniciada ya la "guerra contra el narcotráfico» lanzada por el presidente Felipe Calderón, Chihuahua ocupa el primer lugar en esta tasa seguido de Sinaloa y Durango. Baja California aparece en el doceavo lugar y Tamaulipas en el treceavo. En 2009, Chihuahua conserva el primer lugar seguido de Durango y Sinaloa. Sonora aparece en el octavo puesto y Tamaulipas queda en el onceavo. En 2010, Sinaloa desbanca a Chihuahua y el tercer puesto lo conserva Durango y Sonora queda en noveno lugar, Tamaulipas en décimo, Nuevo León en treceavo y Baja California en el catorceavo. A partir de 2008, los estados de la frontera norte se van acercando a los primeros diez lugares en la tasa de muerte por homicidio, sugiriendo que la guerra contra el narcotráfico ha cobrado un mayor número de víctimas en la región fronteriza del norte de México.

Estos datos muestran que los homicidios causados por la violencia han impactado en las decisiones de posibles inmigrantes internos que prefieren quedarse en sus estados de origen en lugar de arriesgarse a perder la vida en el intento de cambiar su residencia, así como en las decisiones de los residentes de los estados «violentos» que se deciden a emigrar.

Cambios en las tasas de inmigración y emigración en los estados de la frontera norte de México (1995 y 2010)

Tanto la migración interna como la internacional fueron componentes fundamentales que propiciaron el crecimiento acelerado de las poblaciones

$70 \frac{\text { PRIMER SEMESTRE } 2014}{\text { MIGRACIÓN Y DESARROLLO NÚM. } 22}$ 
fronterizas del norte de México desde mediados del siglo xx (Ham, 1992). A pesar de que las crisis económicas experimentadas por el resto del país han detenido la expansión del empleo, en esta región las actividades económicas relacionadas con la industria maquiladora, el turismo y el empleo de las personas que trabajan en Estados Unidos pero viven en ciudades fronterizas mexicanas ("transmigrantes» o "conmuters») logran mantener la capacidad de empleo y retener a los inmigrantes del interior del país. La razón del mejor desempeño de las ciudades fronterizas en relación al resto de México se debe a las diferencias de las economías entre México y Estados Unidos (Alegría, 2010).

El rápido crecimiento poblacional de fines del siglo pasado contribuyó también al ensanchamiento del grupo en edades laborales (Canales, 1999). Esa capacidad de crecimiento demográfico se sostuvo hasta los albores del siglo XXI, a partir del desarrollo comercial, los servicios turísticos, los convenios bilaterales de migración y la localización de la industria manufacturera de exportación (Estrella, 1999).

La frontera norte de México fue, durante largo tiempo, una zona de atracción migratoria, no sólo por la presencia de mayores niveles de desarrollo social (Conapo, 2000) y por sus características económicas, entre las que se encuentra una mayor oferta de empleo, sino también por la cercanía con Estados Unidos. Esto tuvo efectos positivos en la inmigración de personas que buscaban trabajo y mejores condiciones de vida, así como en la atracción de migrantes que iban de paso hacia Estados Unidos, muchos de ellos indocumentados, o bien, de mexicanos que retornaban después de haber pasado una temporada en Estados Unidos con la intención de establecerse en México (Corona, 1991).

Durante el periodo 1995-2010 hubo cambios notables en los niveles de las tasas de inmigración, de emigración y la tasa neta de migración para los tres quinquenios bajo estudio en los seis estados fronterizos. El cuadro 1 contiene la información para los tres quinquenios bajo estudio: 1995-2000, 2000-2005 y 2005-2010, con datos de los conteos de población de 1995 y de 2005 y los censos de población y vivienda de 2000 y de 2010 del INEGI. 


\section{CUADRO 1}

Tasas de inmigración, de emigración y neta de los estados de la frontera norte de México, 1995-2010.

\begin{tabular}{|c|c|c|c|c|c|c|c|c|c|}
\hline \multirow{2}{*}{$\begin{array}{c}\text { PERIODO } \\
\text { ENTIDAD FEDERATIVA }\end{array}$} & \multicolumn{3}{|c|}{$1995-2000$} & \multicolumn{3}{|c|}{$2000-2005$} & \multicolumn{3}{|c|}{ 2005-2010 } \\
\hline & $\mathrm{TI}$ & $\mathrm{TE}$ & TNM & $\mathrm{TI}$ & $\mathrm{TE}$ & TNM & $\mathrm{TI}$ & $\mathrm{TE}$ & TNM \\
\hline Baja California & 21.23 & 6.10 & 15.13 & 12.86 & 4.14 & 8.72 & 11.57 & 9.55 & 2.02 \\
\hline Coahuila de Zaragoza & 6.99 & 6.68 & 0.31 & 3.62 & 3.67 & -0.05 & 5.68 & 5.79 & -0.11 \\
\hline Chihuahua & 10.92 & 3.66 & 7.26 & 4.29 & 2.76 & 1.53 & 4.55 & 6.30 & -1.75 \\
\hline Nuevo León & 7.46 & 4.06 & 3.40 & 4.54 & 2.52 & 2.02 & 8.89 & 4.11 & 4.78 \\
\hline Sonora & 7.69 & 5.98 & 1.71 & 4.17 & 3.95 & 0.22 & 6.09 & 5.46 & 0.63 \\
\hline Tamaulipas & 12.95 & 5.87 & 7.08 & 7.84 & 3.51 & 4.32 & 9.80 & 7.22 & 2.58 \\
\hline
\end{tabular}

$\mathrm{TI}=$ Tasa de Inmigración, $\mathrm{TE}=$ Tasa de Emigración, $\mathrm{TNM}=$ Tasa Neta de Migración

Fuente: Elaboración propia con información de INEGI (2000, 2005 y 2010).

Comparando entre el primer (1995-2000) y tercer quinquenio (2005-2010), las tasas de inmigración muestran consistentemente una tendencia hacia la baja mientras la tasa de emigración ha ido en aumento, lo que tiene como consecuencia una notable disminución en la tasa neta de migración en todos los estados, con excepción de Nuevo León, el único estado que ha incrementado la inmigración y mantenido la emigración, lo que provocó un aumento en la tasa de migración neta.

Mientras que para el quinquenio 1995-2000 las tasas de inmigración de los estados de Baja California, Tamaulipas y Chihuahua se encontraban por arriba de 10.00 por 100 mil habitantes, durante el periodo 2005-2010 solamente Baja California se mantiene por encima de esta cifra. En 1995-2000, la tasa más alta de emigración la tenía Coahuila con 6.99 por 100 mil habitantes y para 2005-2010 es Baja California con 9.55 por mil habitantes.

La tasa neta de migración más elevada la tiene Baja California con 15.15 por mil habitantes en 1995-2000. En 2005-2010 es Nuevo León el estado con la tasa neta de migración más elevada con 4.78 por mil habitantes. Estas cifras revelan la caída que se ha tenido en estos cinco lustros respecto al volumen de población que atraían las entidades del norte del país. Coahuila y Chihuahua muestran incluso tasas netas negativas, destacándose este último estado que pasó de ser el segundo lugar de inmigración de los estados

$72 \frac{\text { PRIMER SEMESTRE } 2014}{\text { MIGRACIÓN Y DESARROLLO NÚM. } 22}$ 
fronterizos durante 1995-2000 con una muy alta tasa neta de migración de 7.26 por mil habitantes a -1.75 por mil habitantes en 2005-2010.

La inmigración ha sido un factor fundamental del crecimiento de la región del norte de México. Ha sido, sin duda, el principal motor de la dinámica poblacional durante los últimos 40 años, sin embargo, durante el periodo analizado (1995-2010), ha disminuido su ritmo e incluso ha caído con respecto a lo que se tenía durante la década de 1990. Ahora factores como el desempleo y la violencia e inseguridad pública han cobrado importancia en cuanto a los efectos en el crecimiento de dichos estados.

El crecimiento de la población y sus tendencias determinan a la estructura por edad y ésta, a su vez, las necesidades en cuanto a infraestructura y servicios que se deben generar para garantizar el bienestar de cada uno de los miembros de la sociedad. En el siguiente apartado se muestran los cambios en las estructuras por edad de la población para 2000, 2005 y 2010, en los que se vislumbran los efectos de los cambios en los patrones de migración.

Con los elementos revisados hasta aquí, aún queda por ver el posible efecto que ha desempeñado el retorno voluntario y la repatriación de mexicanos desde Estados Unidos en el crecimiento de la frontera norte de México. A continuación se presenta una descripción de los cambios que ha habido en el número de eventos de repatriación entre 1995 y 2010.

\section{DEPORTADOS A LA ZONA FRONTERIZA del NORTE de México}

Un aspecto sobre el que se puede especular es el impacto que podría tener en el crecimiento de las ciudades fronterizas la población mexicana deportada desde Estados Unidos en los últimos años y que contrarrestaría la disminución del crecimiento poblacional. Si bien resulta importante tomar en cuenta el número de eventos de repatriación dentro de las consideraciones que se hagan respecto del volumen de la población fronteriza, cabe mencionar que muchos de estos deportados vuelven a intentar el cruce hacia 
Estados Unidos, otros deciden regresar a sus lugares de origen en estados no fronterizos $y$, finalmente, una parte de ellos se establecen definitivamente a lo largo de la frontera y son captados en censos y encuestas.

Según el Departamento de Seguridad Nacional (Department of Homeland Security) existen dos tipos de deportados: los «removidos» y los «retornados». Los removidos (removals) se refiere a las expulsiones obligatorias de extranjeros inadmisibles o deportables, con base en una orden de remoción con consecuencias administrativas o penales sobre reingresos subsecuentes a Estados Unidos. El término retornados (returns) hace referencia a las expulsiones de extranjeros inadmisibles o deportables, que no se fundamentan en una orden de remoción. La mayor parte de estos retornos voluntarios son de ciudadanos mexicanos que han sido aprehendidos por la Patrulla Fronteriza (Border Patrol) y deportados a México (us Department of Homeland Security, 2011; Alarcón y Becerra, 2012). La gráfica 3 muestra la información de los repatriados entre 1995 y 2010 del Instituto Nacional de Migración (INM) con base en la información registrada en los puntos oficiales de repatriación sin hacer la distinción entre los que fueron removidos o retornados.

\section{GRÁFICA 3}

Eventos de repatriación de Estados Unidos a estados de la frontera norte, 1995-2010

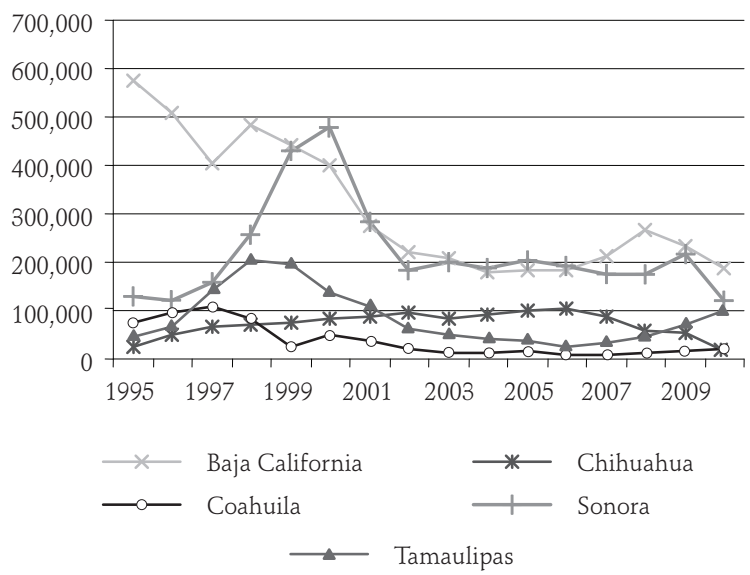

Fuente: INM (2010).

$74 \frac{\text { PRIMER SEMESTRE } 2014}{\text { MIGRACIÓN Y DESARROLLO NÚM. } 22}$ 
El comportamiento de los eventos de deportación ha variado en el tiempo y cada estado muestra tendencias diferentes, como lo muestra la gráfica 3. Sonora, que se ha convertido en el principal lugar de cruce indocumentado en los últimos años, sustituyendo a Baja California, muestra a partir de 1998 un aumento muy notorio al llegar casi a 500 mil eventos en 2000, para luego disminuir en 2003 y mantenerse alrededor de 200 mil hasta 2009 y tener nuevamente una caída en 2010 con un poco mas de 100 mil eventos registrados para ese año. Sonora registra más que otra cosa a los retornados por las autoridades migratorias de Estados Unidos en el intento de cruzar de forma indocumentada hacia ese país.

En Baja California, el número de deportados comienza con cerca de 600 mil eventos en 1995 y disminuye a alrededor de 400 mil en 1997 y se mantiene muy bajo el resto del periodo ubicándose en cerca de 200 mil eventos anuales con un importante incremento en 2007, que puede deberse al mayor número de removidos desde el interior de Estados Unidos. Tamaulipas muestra un incremento notable entre 1998 y 2000 (200 mil eventos aproximadamente durante esos dos años) y se mantiene por debajo de 100 mil durante el resto del periodo, incrementado nuevamente el número de eventos en 2010. Chihuahua permanece por debajo de 100 mil eventos durante todo el periodo de observación, y Coahuila solamente hasta 1998 se encuentra cercano a esta cifra y el resto del periodo se mantiene inferior a 50 mil y cercano apenas a 10 mil.

En 2010, de acuerdo a datos del INM, hubo 469,268 eventos de repatriación de nacionales mexicanos de Estados Unidos. Del número total de repatriaciones, 133,770 (28\%) se llevaron a cabo por Tijuana, la ciudad que recibió el mayor número de migrantes mexicanos deportados en 2010, con un promedio de 366 eventos de repatriación diarios. Las repatriaciones en 2010 se dieron a través de ciudades de los estados fronterizos de Baja California, Sonora, Coahuila, Chihuahua y Tamaulipas, además del aeropuerto internacional de la ciudad de México (Alarcón y Becerra, 2012).

La deportación y el retorno voluntario de mexicanos desde Estados Unidos durante el último quinquenio puede captarse también a través del censo de 2010 con la pregunta sobre residencia en 2005 de la población mayor de 


\section{CUADRO 2}

Proporción de población mayor de 12 años por entidad federativa que declaró ser residente en Estados Unidos en 2005 según orden jerárquico de la distribución de los hombres

\begin{tabular}{|c|c|c|c|c|}
\hline ORDEN JERÁRQUUICO & ENTIDAD & TOTAL & HOMBRES & MUJERES \\
\hline 1 & Zacatecas & 2.0 & 3.1 & 0.9 \\
\hline 2 & Michoacán de Ocampo & 1.8 & 2.7 & 0.9 \\
\hline 3 & Nayarit & 1.8 & 2.5 & 1.2 \\
\hline 4 & Guanajuato & 1.3 & 2.2 & 0.5 \\
\hline 5 & Hidalgo & 1.3 & 2.1 & 0.6 \\
\hline 6 & Colima & 1.6 & 2.1 & 1.1 \\
\hline 7 & Baja California & 1.5 & 2.0 & 1.1 \\
\hline 8 & Morelos & 1.2 & 1.7 & 0.7 \\
\hline 9 & Aguascalientes & 1.1 & 1.6 & 0.6 \\
\hline 10 & Durango & 1.1 & 1.6 & 0.7 \\
\hline 11 & Guerrero & 1.0 & 1.6 & 0.5 \\
\hline 12 & Oaxaca & 1.0 & 1.6 & 0.5 \\
\hline 13 & Jalisco & 1.1 & 1.6 & 0.7 \\
\hline 14 & San Luis Potosí & 1.0 & 1.6 & 0.4 \\
\hline 15 & Sonora & 1.2 & 1.5 & 0.9 \\
\hline 16 & Querétaro & 0.8 & 1.4 & 0.3 \\
\hline 17 & Chihuahua & 1.0 & 1.3 & 0.8 \\
\hline 18 & Veracruz & 0.7 & 1.1 & 0.3 \\
\hline 19 & Puebla & 0.6 & 1.0 & 0.3 \\
\hline 20 & Tamaulipas & 0.7 & 0.9 & 0.5 \\
\hline 21 & Baja California Sur & 0.7 & 0.9 & 0.5 \\
\hline 22 & Sinaloa & 0.7 & 0.9 & 0.5 \\
\hline 23 & Tlaxcala & 0.5 & 0.8 & 0.2 \\
\hline 24 & Coahuila de Zaragoza & 0.5 & 0.6 & 0.3 \\
\hline 25 & Quintana Roo & 0.4 & 0.5 & 0.3 \\
\hline 26 & Campeche & 0.3 & 0.5 & 0.1 \\
\hline 27 & México & 0.3 & 0.5 & 0.2 \\
\hline 28 & Chiapas & 0.3 & 0.5 & 0.1 \\
\hline 29 & Nuevo León & 0.3 & 0.4 & 0.2 \\
\hline 30 & Yucatán & 0.2 & 0.4 & 0.1 \\
\hline 31 & Distrito Federal & 0.2 & 0.3 & 0.1 \\
\hline 32 & Tabasco & 0.1 & 0.2 & 0.1 \\
\hline
\end{tabular}

Fuente: INEGI (2010). 
12 años. El cuadro 2 contiene la información sobre la distribución por entidad federativa de la proporción de los que fueron residentes en Estados Unidos en 2005. El orden jerárquico es según la proporción de los hombres debido a que la migración sigue siendo un fenómeno predominantemente masculino.

Los primeros seis lugares, de acuerdo al porcentaje de varones mayores de 12 años que declararon haber sido residentes en Estados Unidos en 2005, los ocupan los estados de la región tradicional de la migración a Estados Unidos, además del estado de Hidalgo. Baja California está dentro de los primeros 10 lugares, lo que resulta comprensible debido a que comparte la frontera con California, que es el estado que tiene la mayor concentración de población inmigrante mexicana. En 2005, el primer lugar de las entidades federativas lo ocupa Zacatecas con 3.1\% del total de hombres con residencia en Estados Unidos, seguido de Michoacán con 2.7\% y Nayarit con 2.5\%.

Aparte de Baja California, del resto de los cinco estados de la frontera norte de México, tres se encuentran entre el lugar 15 y 20 (Sonora, 1.5\%; Chihuahua, 1.3\%, y Tamaulipas, 0.9\%) y dos antes del 30 (Coahuila, 0.6\%, y Nuevo León, $0.4 \%$ ).

Esta distribución permite plantear la hipótesis de que tanto los deportados (removidos y retornados) como los que regresaron voluntariamente, en su mayoría, regresan a sus estados de origen y pocos son los que permanecen en los estados fronterizos. Sin embargo, de continuar la política agresiva de Estados Unidos que busca deportar a la mayor parte de los inmigrantes indocumentados mexicanos que residen en Estados Unidos bajo la acusación de que son criminales, podemos esperar que muchos de ellos se establecerán en las ciudades fronterizas de Baja California. Entre estos deportados se puede esperar que los que han sido separados de sus familias buscarán establecerse en ciudades como Tijuana o Mexicali para tratar de seguir en contacto con sus cónyuges e hijos que sean residentes permanentes legales o ciudadanos de Estados Unidos que puedan cruzar la frontera para visitarlos.

Las opciones de los migrantes deportados dependen mucho de las políticas y apoyos que el gobierno mexicano implemente en las ciudades fronterizas para que se incorporen a la sociedad y al mercado laboral. En este sentido, los deportados que fueron llevados por sus padres a Estados Unidos 
cuando eran niños, los asíllamados dreamers, presentan necesidades especiales ya que muchos de ellos nunca regresaron a México después del cruce indocumentado, no hablan bien español y sus familiares cercanos viven en Estados Unidos. El programa de Repatriación Humana del gobierno federal, que prácticamente no tiene presupuesto, hasta este momento no ofrece opciones viables para esta población (Alarcón y Becerra, 2012).

\section{Cambios demográficos en los estados DE LA FRONTERA NORTE DE MÉXICO, 1995-2010}

El crecimiento de una población puede conocerse mediante la descomposición de las variaciones numéricas de una población en un determinado intervalo de tiempo en sus cuatro componentes: nacimientos, defunciones, inmigraciones y emigraciones. Estos cuatro componentes determinan el crecimiento total de una población. A la diferencia entre los nacimientos y las defunciones se le conoce como crecimiento natural o saldo natural y a la diferencia entre inmigraciones y emigraciones como crecimiento social o saldo neto migratorio (Livi Bacci, 2007).

La primera característica demográfica que distinguía a los estados de la frontera norte de México durante la década de 1990 era su gran crecimiento poblacional comparado con el resto del país. Una forma de medir y comparar el crecimiento total de una población es mediante el cálculo de la tasa anual de crecimiento. El cuadro 3 muestra las tasas de crecimiento para México y para cada uno de los estados que colindan con Estados Unidos para los periodos 1990-1995, 1995-2000, 2000-2005 y 2005-2010. Lo primero que destaca es que todas las tasas de crecimiento, tanto de México como de los estados de la frontera norte disminuyen consistentemente durante los tres primeros quinquenios entre 1990 y 2005. En el primer periodo (1990-1995) se puede observar que los estados de la frontera norte crecían a una tasa de 2.5\% anual mientras que México lo hacía a 2.1\% anual. Destaca Baja California entre los estados con la mayor tasa anual de crecimiento de $4.3 \%$ anual y Coahuila con la más baja de $1.7 \%$ anual. 
En el periodo 1995-2000, el crecimiento de los estados de la frontera norte fue de $2.1 \%$ anual, muy por arriba de la tasa nacional de $1.6 \%$. En el primer quinquenio de este siglo (2000-2005), si bien la tasa disminuye, la diferencia se mantiene. Mientras los estados fronterizos alcanzaron una tasa de 1.6\% anual, la del país fue de 1.0\%. Sin embargo, en el periodo 2005 2010, la tasa de los estados de la frontera norte de México se incrementa a un ritmo más lento con respecto al periodo anterior, llegando solamente a $1.9 \%$ anual mientras que la tasa anual del país sube a 1.8\% (INEGI, 2002, 2005 y 2010).

\section{CUADRO 3}

Tasas de crecimiento anuales de la población de los estados fronterizos y de México 1990-2010

\begin{tabular}{lcccc}
\hline & $1990-1995$ & $1995-2000$ & $2000-2005$ & $2005-2010$ \\
\hline Estados Unidos Mexicanos & 2.1 & 1.6 & 1.0 & 1.8 \\
Frontera Norte & 2.5 & 2.1 & 1.6 & 1.9 \\
Baja California & 4.3 & 3.9 & 2.4 & 2.2 \\
Coahuila de Zaragoza & 1.7 & 1.3 & 1.5 & 2.0 \\
Chihuahua & 2.4 & 2.1 & 1.1 & 1.0 \\
Nuevo León & 2.4 & 1.8 & 1.6 & 2.1 \\
Sonora & 2.4 & 1.4 & 1.4 & 2.1 \\
Tamaulipas & 2.1 & 2.0 & 1.7 & 1.6 \\
\hline
\end{tabular}

Fuente: INEGI $(1996,2000,2010,2006)$.

Las tasas de crecimiento disminuyen consistentemente en todos los estados fronterizos durante los tres primeros quinquenios entre 1990 y 2005. Durante el cuarto periodo, entre 2005 y 2010, se pueden observar dos tipos de comportamiento. Por una parte, en los estados de Coahuila, Sonora y Nuevo León la tasa de crecimiento se recupera entre 2005 y 2010, el mismo comportamiento que se observa en el país. Sin embargo en Baja California, Chihuahua y Tamaulipas la caída en la tasa de crecimiento es notable en el periodo 2000-2005.

Estos cambios en la dinámica del crecimiento poblacional de los estados de la frontera norte tienen que ver, por una parte, con los cambios mismos del 
crecimiento natural de la población, como la disminución de la fecundidad. Por otra parte, estos cambios se pueden deber a los patrones de movilidad interna de la población causados tanto por la percepción de una disminución de las oportunidades económicas como por la percepción de la inseguridad y de la violencia que se vive en la frontera norte. Si bien, el crecimiento de décadas anteriores ha disminuido tanto en el país como en los estados de la frontera norte, el efecto en la estructura por edad y sexo ha sido distinto y las causas también lo son. A continuación se describen los principales cambios en las estructuras de la población por edad.

Los cambios en las estructuras de población por edad

Los cambios que se dan en los componentes demográficos (mortalidad, fecundidad y migración) tienen un efecto distinto en la estructura por edad de la población. Estos cambios pueden visualizarse a través de las pirámides de población que permiten observar cómo el crecimiento distinto de los estados genera diferentes estructuras por edad y sexo, según la entidad federativa de que se trate. Los efectos de los cambios en la natalidad se aprecian en los grupo 0-4 y 5-9 y la inmigración y la emigración en los grupos de edades jóvenes, 20-24, 25-29 y 30-34, según el tamaño de los escalones de la pirámide. La mortalidad, si no presenta cambios muy fuertes por el incremento de homicidios, accidentes, epidemias o eventos catastróficos, no afecta la estructura de la pirámide y solamente se nota el incremento de los mayores de 60 años al incrementarse la esperanza de vida.

La gráfica 4 muestra la estructura porcentual de la población por grupo quinquenal de edad y por sexo para los seis estados de la frontera norte de México según datos del censo de 1990.

De acuerdo con la gráfica 4, con excepción de Baja California y Chihuahua, el resto de los estados muestra una clara disminución de los grupos 0-4 y 5-9, como efecto de la disminución de la fecundidad que se observaba ya desde la década de 1980. Como estados de atracción de inmigrantes durante este periodo, tanto Baja California como Chihuahua muestran una proporción mayor de jóvenes en edades reproductivas y laborales por lo que

$$
80 \frac{\text { PRIMER SEMESTRE } 2014}{\text { MIGRACIÓN Y DESARROLLO NÚM. } 22}
$$



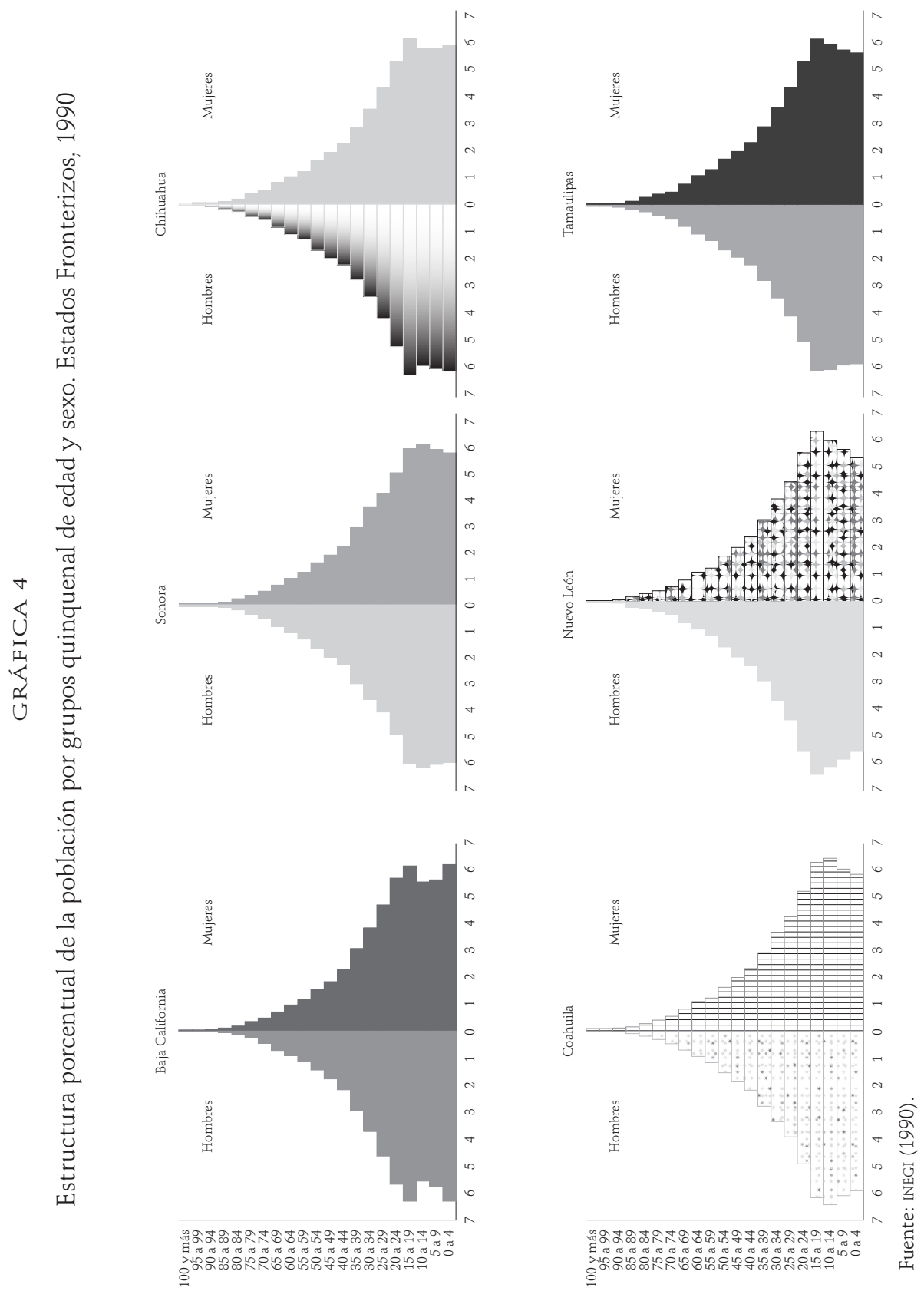
el número de nacimientos también es mayor, a pesar de que también la fecundidad había descendido para este periodo en dichas entidades.

Para 2000, los efectos de la disminución de la fecundidad y del inicio del descenso del crecimiento por inmigración ya son evidentes en las estructuras por edad de los seis estados, como se puede observar en la gráfica 5 . La base se ensancha para los primeros dos grupos de edad y pudiera deberse a un incremento en la fecundidad durante la década de los noventa para tales estados. Esta forma inicial de las pirámides de los seis estados fronterizos muestra una capacidad importante de crecimiento debido a la muy alta concentración de la población en edades productivas y reproductivas. Son estados en los que para 2000 se encontraban en un relativo auge económico y de bienestar social que invitaba a la población del resto del país a participar de dicho bienestar y por eso las altas tasas de inmigración que se aprecian durante la década de 1990.

El dinámico crecimiento poblacional de Baja California (tasas de crecimiento anual de $4.3 \%$ en $1990-1995$ y 3.9\% en 1995-2000) se refleja claramente en la pirámide de 2000. Destaca la magnitud de los grupos 0-4, 5-9 por la base y el 20-24, 25-29 en las edades productivas y reproductivas. Nuevo León presenta la misma forma que Baja California. Los estados de Sonora, Chihuahua y Coahuila comienzan a mostrar los efectos de la continua disminución de la fecundidad, los escalones de los grupos 0-4 y 5-9 son muy parecidos. En Tamaulipas se observa un aumento en el grupo 20-24, pero únicamente para las mujeres y también solamente para ellas, una disminución en el 10-14.

En 2010, la gráfica 6 muestra las tasas de crecimiento a la baja en todos los estados fronterizos y una tasa de emigración en aumento. La forma de las pirámides muestran los efectos de estos dos factores tanto en la base como en los grupos de jóvenes. En primer lugar, es clara la disminución de la base de la pirámide de edad ya que el primer escalón, el del grupo 0-4, es menor que el del grupo 5-9 en los seis estados. Este primer aspecto indica que el efecto de la inmigración en edades reproductivas ya no tiene el impacto en el número de nacimientos como lo tuvo en periodos anteriores. Esta es la primera vez que se nota el efecto de la disminución de la fecundidad en las pirámides de edades de los estados fronterizos.

$82 \frac{\text { PRIMER SEMESTRE } 2014}{\text { MIGRACIÓN Y DESARROLLO NÚM. } 22}$ 

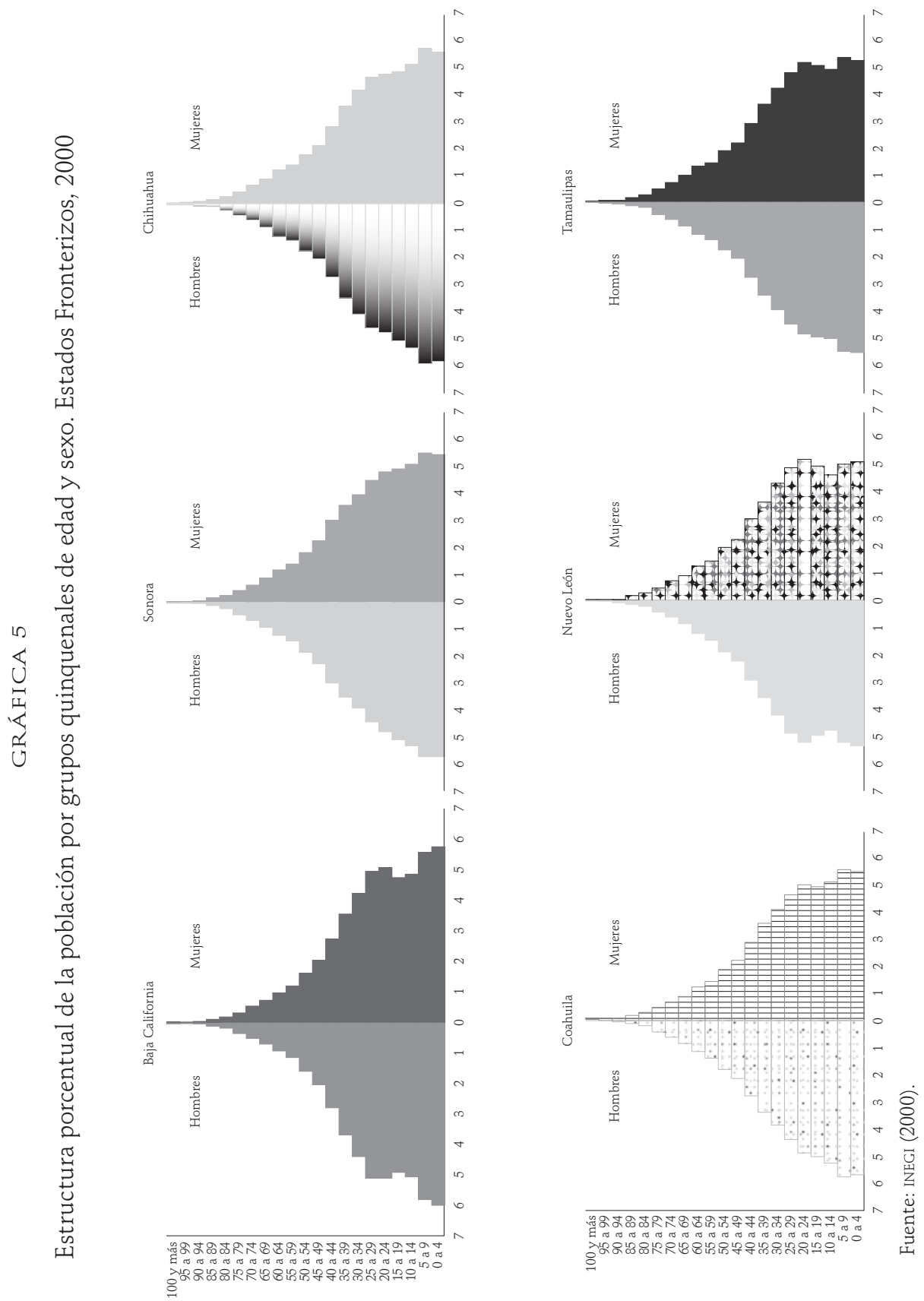
Con respecto a los grupos de edades correspondientes a los jóvenes, sobresale el estado de Chihuahua, ya que muestra los inicios de una «salida» del estado de personas en las edades productivas y reproductivas. Se observa una disminución en los escalones correspondientes a los grupos de edad 2024 y 25-29 comparando con el 30-34, tanto para hombres como para mujeres. En Sonora puede apreciarse el mismo efecto, pero con menos fuerza que en Chihuahua y con mayor énfasis del lado masculino. Este aspecto es un primer indicio de las transformaciones en la estructura por edad de la población debido a los cambios en la inmigración y en la emigración hacia y desde los estados de la frontera norte de México.

Baja California y Nuevo León van conformando su estructura en un "pilar» al mostrar en cada escalón de la pirámide un tamaño similar al anterior desde el grupo 0-4 hasta el 30-34. En Coahuila pueden verse más los efectos de una disminución de la fecundidad en el grupo 0-4 y también el efecto de una menor inmigración de personas en edades productivas. Tamaulipas aún conserva rasgos de una forma de pirámide pero cada vez con una base que va disminuyendo su importancia relativa respecto del resto de grupos de edad.

Estos cambios muestran la capacidad de cambio en la estructura por edad de la población que tiene un fenómeno demográfico como la inmigración/emigración en el corto plazo. La disminución de la inmigración respecto a lo que se había experimentado anteriormente en la región ha detenido el efecto de rejuvenecimiento poblacional que se había dado durante aproximadamente 25 años (Ybáñez y Alarcón, 2007).

El impacto que tiene el cambio de la inmigración/emigración en la estructura por edad de los estados de la frontera norte puede apreciarse en la gráfica 6. En tan sólo diez años la forma de las pirámides muestra los efectos del incremento de la emigración en el último lustro, sobre todo en los estados de Chihuahua, Sonora y Tamaulipas que tienen la mayor inseguridad y la mayor tasa de homicidios durante la última década.

En los dos primeros quinquenios del siglo xxI, el crecimiento social ha disminuido su intensidad en las entidades de la frontera norte de México, las estructuras por edad de las pirámides muestran los efectos de una emi-

$$
84 \frac{\text { PRIMER SEMESTRE } 2014}{\text { MIGRACIÓN Y DESARROLLO NÚM. } 22}
$$


gración más pronunciada en estados como Chihuahua, Coahuila y Sonora, y aún incipiente en Baja California y Tamaulipas.

Por otra parte, Nuevo León es el estado que todavía muestra un crecimiento social importante en comparación con las otras cinco entidades. El dinamismo poblacional que se tenía en las entidades del norte a fines del siglo pasado dejó de observarse en los inicios del siglo xxi. Esta situación tendrá efectos en el futuro envejecimiento de la región ya que, si bien la inmigración ha frenado el proceso de envejecimiento de esta parte del país, en las próximas décadas éste se acelerará tanto por la disminución de la fecundidad como por el número de personas que se encuentran en los grupos de 45 años y más, que seguirán contribuyendo al incremento futuro de los mayores de 60 años.

Todos los cambios descritos con anterioridad van a tener efecto en el futuro envejecimiento demográfico de la región debido a que el descenso de la inmigración de jóvenes que desaceleraba el proceso de envejecimiento ya no va a jugar ese papel tan importante $y$, al contrario, al estar aumentando la emigración de población en edades productivas y reproductivas, lo que probablemente suceda es un aceleramiento en el proceso de envejecimiento poblacional.

Las posibles causas de los cambios en el crecimiento social tiene dos vertientes. Por una parte, el crecimiento del desempleo en los estados la frontera norte es un factor que disminuye la atracción hacia estas entidades, por otra parte, el incremento de la tasa de homicidios que genera una percepción de inseguridad fomenta la emigración desde estas entidades.

\section{CONCLUSIÓN}

El gran crecimiento poblacional de los estados de la frontera norte de México que culminó al final del siglo xx se debió principalmente a la gigantesca inmigración interna. Este proceso fue seguido de la disminución en el ritmo de crecimiento poblacional que ha resultado de la combinación de la reducción 

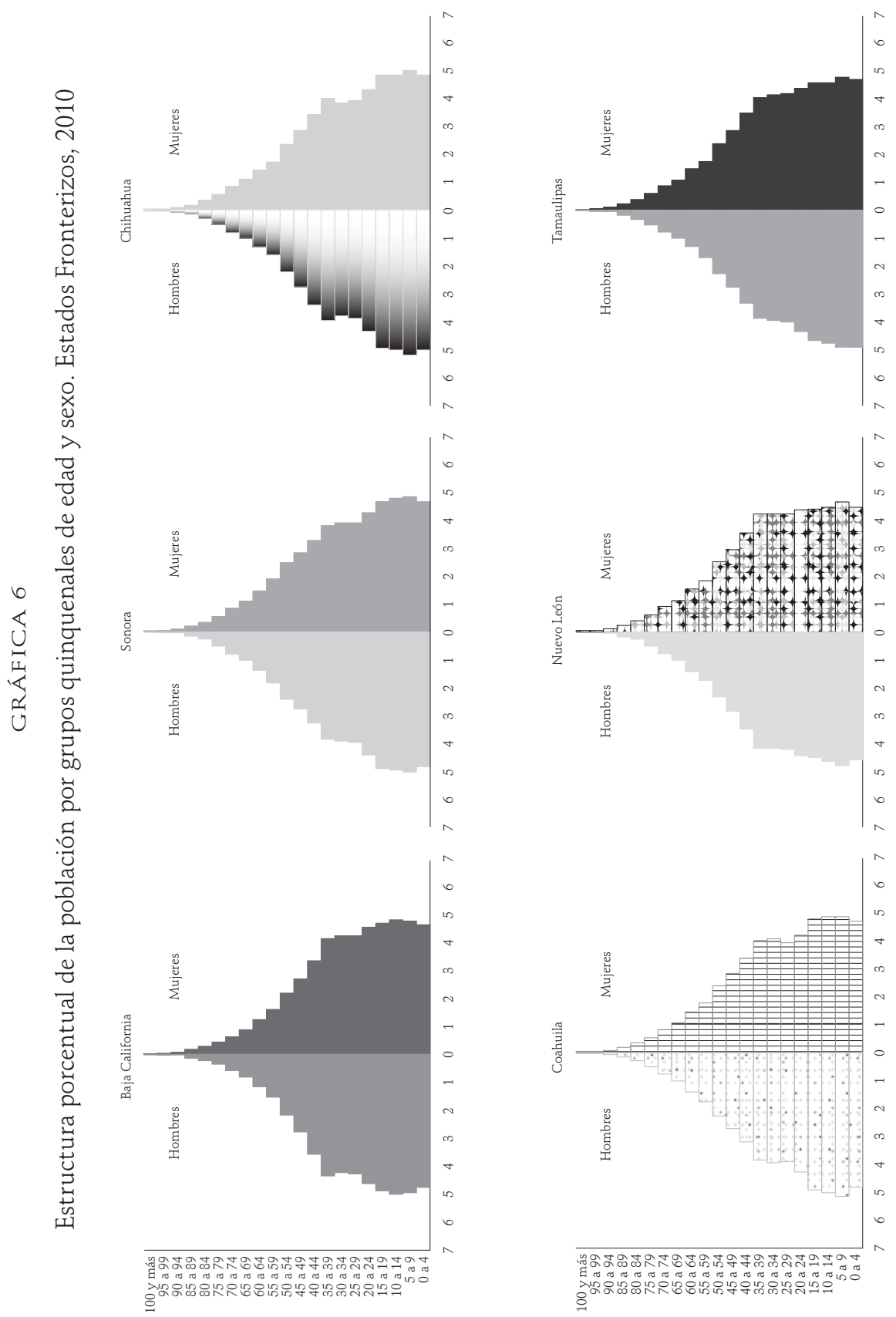

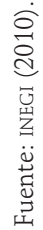


de la inmigración y el incremento de la emigración que se observa claramente en la primera década del siglo XXI.

En este sentido, la transformación de la dinámica poblacional de los estados de la frontera norte tiene que ver, por una parte, con los cambios del crecimiento natural de la población, como la disminución de la fecundidad, y, por otra parte, con los cambios en los patrones de inmigración y emigración interna de la población causados tanto por la percepción de inseguridad y violencia en la frontera norte como por la percepción de una disminución de las posibilidades económicas debido a la caída de las oportunidades de empleo en la región. Los estados de la frontera norte de México parece ser que han dejado de ser el polo de atracción más dinámico del país.

La percepción de inseguridad y violencia en los estados de la frontera norte de México ha impactado la movilidad de las personas afectando la tasa de crecimiento durante la última década, con consecuencias visibles en la estructura por edad de las pirámides de 2010, especialmente en estados como Chihuahua y Tamaulipas. El alto consumo de drogas, la violencia juvenil, el tráfico de armas en la frontera, la alta movilidad poblacional, la disparidad socioeconómica, las disputas políticas y la ausencia de una cultura de la legalidad han llevado a que muchas mujeres y niñas sean blanco idóneo de esta violencia estructural regional.

Sin embargo, además de estos factores, el distinto dinamismo económico de los estados contiguos de Estados Unidos también juega un papel fundamental en el desarrollo población de los estados de la frontera norte de México. Es muy diferente la economía del estado de California que la de Texas (Ybáñez y Alarcón, 2007), por esta razón, la tasa de desempleo de Baja California se mantuvo por debajo hasta 2009 con relación a otros estados fronterizos. Lo anterior también tiene impactos en la estructura por edad de los seis estados y en el menor grado de envejecimiento que mostrará esta entidad.

De continuar las tendencias del último quinquenio, el futuro de la estructura por edad de la población de los estados fronterizos podría mostrar señales de un envejecimiento más acelerado que el previsto por las proyecciones demográficas realizadas por el Conapo. El efecto positivo de la inmigración de jóvenes que detenía el proceso de envejecimiento en la región ha 
dejado de ser la opción en las entidades norteñas debido no sólo a la disminución de la misma sino también al efecto de la emigración en edades productivas y reproductivas desde estos mismos estados.

Si bien es cierto que la proporción de mayores de 65 años en los estados de la frontera norte se mantendrá por debajo de lo esperado para el resto de México, cabe destacar que las diferencias entre los estados fronterizos deben de ser considerados a la hora de planificar los recursos que se destinarán a la infraestructura de servicios públicos y de salud. Las demandas de la población en edades productivas y reproductivas son distintas de las de los mayores de 65 años.

Más que en ninguna otra región, en la frontera norte de México es innegable la necesidad de considerar la demografía dentro de las políticas de desarrollo regional, que es la base para el diseño de políticas públicas sobre educación, seguridad o salud. La inercia demográfica obliga a planear a largo plazo, pues los fenómenos demográficos imprimen sus huellas en la pirámide de edades las cuales tienen repercusiones a lo largo de muchas décadas.

\section{Bibliografía}

Alarcón, Rafael y William Becerra (2012), «żCriminales o Victimas ? La deportación de migrantes mexicanos de Estados Unidos a Tijuana, Baja California», Norteamérica. año 7 , núm. 1.

y Luis Enrique Calva Sánchez (2013), «La Emif Norte y la deportación de migrantes mexicanos a Estados Unidos (1999-2012)», El Colegio de la Frontera Norte.

Alegría, Tito (2010), «Estructura de las ciudades de la frontera norte», en Gustavo Garza y Martha Schteingart (coords.), Desarrollo urbano y regional. Los grandes problemas de México (vol. 2), México, El Colegio de México.

Calderón Villarreal, Cuauhtémoc (2009), «Dinámica y tendencias económicas de los estados de la Frontera Norte de México», en Plan indicativo para el desarrollo competitivo y sustentable de la región transfronteriza México-Estados Unidos, xxvi Conferencia de gobernadores fronterizos, Monterrey.

$88 \frac{\text { PRIMER SEMESTRE } 2014}{\text { MIGRACIÓN Y DESARROLLO NÚM. } 22}$ 
Canales Cerón, Alejandro (1999), «Migración y urbanización en la frontera norte de México", en Gabriel Estrella V., Alejandro Canales Cerón A., y María Eugenia Zavala de Cosío, Ciudades de la Frontera Norte: migración y fecundidad. Mexicali, Universidad Autónoma de Baja California.

Chávez Galindo, Ana María y Fernando Lozano Ascencio (2004), "La migración interna en México en el contexto de la globalización: algunas reflexiones», en Fernando Lozano Asencio, El amanecer del siglo y la población mexicana (VI Reunión Nacional de Investigación Demográfica en México) vol. I, Cuernavaca, Morelos, Universidad Nacional Autónoma de México-CRIM-Sociedad Mexicana de Demográfica.

Conapo (2002), Proyecciones de población por municipio 2000-2030, México.

Corona Vázouez, Rodolfo (1991), «Principales características demográficas de la zona fronteriza del norte de México", Frontera Norte, vol. 3, núm. 5.

Escalante, G. Fernando (2009), «Homicidios 1990-2007», Nexos, vol. xxxi, núm. 381. Estrella Valenzuela, Gabriel, Alejandro Canales Cerón y María Eugenia Zavala de Cosío (1999), Ciudades de la Frontera Norte: migración y fecundidad, Universidad Autónoma de Baja California, Mexicali.

Ham Chande, Roberto (1992), "Diferenciales de población y migración internacional a corta distancia en la frontera México-Estados Unidos», Frontera y Migraciones, vol. III.

Hernández Bringas, Héctor H. (1989), Las muertes violentas en México, Centro Regional de Investigaciones Multidisciplinarias, México.

Hans, P. Johnson (1996) Undocumented Immigration to California: 1980-1993, San Francisco, http://www.ppic.org/publications/PPIC100/index.html.

Instituto NaCional De Migración (INM) (2010), «Eventos de repatriación de mexicanos desde Estados Unidos, según entidad federativa y punto de repatriación, 1995-2010», en http://www.inm.gob.mx/index.php/page/Series_Historicas (consultado el 29 de agosto de 2011).

INEGI (2002), XII Censo General de Población y Vivienda 2000, INEGI, Aguascalientes. INEGI (2006), II Conteo General de Población y Vivienda 2005, INEGI, Aguascalientes.

INEGI (2011), XIII Censo General de Población y Vivienda 2010, InEGI, Aguascalientes. INEGI (2011), Comunicado número 287/11, Homicidios por cada 100 mil habitantes por entidad federativa según año de registro, 28 de julio de 2011, Aguascalientes. 
Livi-BACCI, Massimo (2007), Introducción a la demografía, $3^{a}$ ed., Barcelona, Ariel Historia.

Mendoza Cota, Eduardo (2013), "U.S.-Mexican economic integration and its effects on unemploymente in Mexico's northern Border States", Journal of Borderlands Studies, vol 28. núm 1, New Mexico State University, Las Cruces, 2013.

MendozA, Jorge Eduardo (2010), «El mercado laboral en la frontera norte de México: estructura y políticas de empleo», Estudios Fronterizos, Vol. 11, Núm. 21, EneroJunio, pp. 9-42.

Public Policy Institute of California (ppic) (1997), «Return Migration to Mexico: Who Goes? Who Stays?», Research Brief, San Francisco, en http://www.ppic. org/publications/PPICI02/pp102rb.html.

Sistema Nacional de Información en Salud (sinais) (2012), Principales diez causas de mortalidad en México, en http://sinais.salud.gob.mx/descargas/xls/diezprincausasmort2010_CNEGySR.xls (Consultado el 8 de marzo de 2012).

us Department of Homeland Security (DHS) (2011), Yearbook of Immigration Statistics: 2010, Washington, Government Printing Office.

Ybañez Zepeda, Elmyra y Rafael Alarcón (2007), «Envejecimiento y migración en Baja California», Frontera Norte, vol. 19, núm. 38.

Zenteno Quintero, Rene y Rodolfo Cruz Piñeiro (1992), «A Geodemographic Definition of the Northern Border of Mexico", en John R. Weeks y Roberto Ham Chande, Demographic Dynamics of the U.S.-Mexico Border, El Paso, University of Texas. 